\title{
Spectral Efficiency Analysis of Mobile Femtocell Based Cellular Systems
}

\author{
Fourat Haider ${ }^{1}$, Cheng-Xiang Wang ${ }^{1}$, Harald Haas ${ }^{2}$, Dongfeng Yuan ${ }^{3}$, Haiming Wang ${ }^{4}$, Xiqi Gao ${ }^{4}$, Xiao-Hu You ${ }^{4}$, \\ and Erol Hepsaydir ${ }^{5}$ \\ ${ }^{1}$ Joint Research Institute for Signal and Image Processing, Heriot-Watt University, Edinburgh EH14 4AS, UK. \\ ${ }^{2}$ Joint Research Institute for Signal and Image Processing, University of Edinburgh, Edinburgh EH9 3JL, UK. \\ ${ }^{3}$ School of Information Science and Engineering, Shandong University, Jinan, 250100, China. \\ ${ }^{4}$ School of Information Science and Engineering Southeast University, Nanjing, 210096, China. \\ ${ }^{5}$ Hutchison 3G UK, 20 Grenfell Rd., Maidenhead, SL6 1EH, UK. \\ Email: \{fsh12, cheng-xiang.wang\}@hw.ac.uk, h.haas@ed.ac.uk, dfyuan@sdu.edu.cn, \{hmwang, xqgao, xhyu $\} @$ seu.edu.cn, \\ erol.hepsaydir@three.co.uk
}

\begin{abstract}
In this paper, we propose a new concept called mobile Femtocell (MFemtocell) network, which can be considered as a practical implementation of mobile relays (more precisely, moving networks). MFemtocells can be deployed in moving vehicles, such as trains, buses, or private cars to provide enhanced user throughput, extended coverage, and reduction of the signaling overhead and drop calls. We investigate the spectral efficiency of cellular systems with MFemtocell deployment and two resource partitioning schemes. Simulation results demonstrate that with the deployment of MFemtocells, the spectral efficiency and average user throughput can significantly be increased while the signaling overhead is reduced.
\end{abstract}

\section{INTRODUCTION}

Cooperative multi-hop communications with relays can greatly improve the spectral efficiency and network coverage and has widely been considered as a major candidate technology in various standards, such as in Long Term Evoluation (LTE)-Advanced systems. In relay communications, intermediate nodes are used which relay the data to/from the base station (BS) [1]. There are two different types of relays, namely, fixed and mobile relays [2]. Fixed relays are deployed at locations according to cell planning and radio optimization to improve the user throughput or to expand the coverage at the cell edge [3], [4]. Mobile relays [5] are moving wireless nodes which fully support relaying functionalities in cellular systems. Two types of mobile relays can further be distinguished, i.e., mobile user relays and moving networks. Mobile user relays are to use handsets within the close vicinity to relay the information to/from the BS. In moving networks, dedicated relays are mounted on moving vehicles, such as trains, buses, or private cars, to receive data from the BS and forward to the users onboard, and vice versa. In previous works, relay communications mostly focused on scenarios with either fixed relays [6], [7] or mobile user relays [8], [9]. There has been little research undertaken for moving networks.

Public vehicles, e.g., trains and buses, are moving hotspots with many people potentially requesting diverse data services, e.g. web browsing, video streaming, and gaming. Users inside a moving vehicle may execute multiple handovers at the same time causing a significant increase in signaling load and drop connections to the network. So, it is worth minimizing the signaling load and drop calls within a fast moving vehicle.

Femtocells, also called home BSs or home evolved NodeB (Home eNodB) [10], are small, low-power data access points installed by home users to get better indoor coverage or improved user throughput with reduced implementation cost [11]. Femtocells can connect to the operator's core network via legacy broadband connection such as Digital Subscriber Line or optical fiber. Due to the short transmission range between the Femtocell and its users, the transmission power of users can be reduced resulting in longer battery life.

Motivated by the concepts of mobile relays (moving networks) and Femtocells, in this paper we propose a new concept called MFemtocell. There are, however, many challenges for researching MFemtocells, e.g., the way resources are shared by macrocell and MFemtocell users, spectrum reuse strategy under a number of MFemtocel deployments, and spectral and energy efficiencies.

A number of studies have been done on spectrum sharing in Femtocell [12]-[14] and multi-hop [7] in Orthogonal Frequency-Division Multiple Access (OFDMA) based cellular systems. The Femtocell may share the same spectrum with macrocell (i.e., non-orthogonal mode) or utilize a dedicated spectrum (i.e., orthogonal mode) [12]. In an orthogonal spectrum-sharing network, the Femtocell uses a separate spectrum band that is not used by macrocell BSs. This mode can avoid interference to/from the macrocell, i.e., intra-cell interference. However, additional spectrum resources are required and hence the spectral efficiency will be reduced. In this paper we study the impact of two resource partitioning schemes on the spectral efficiency in MFemtocell based LTEAdvanced cellular systems.

The rest of this paper is organized as follows: Section II gives an overview of MFemtocells. Section III presents the system model. Section IV summarizes the simulation assumptions and presents simulation results with detailed analysis. Finally, Section V concludes the paper. 


\section{Mobile Femtocell}

Combining the concept of moving network and Femtocell network, MFemtocell is a small cell which can move around and dynamically change its connection to the operator's core network. MFemtocell can be seen as a practical implementation of moving networks and can be deployed on public transport buses, trains, and private cars. It can adopt a cellular standard (e.g., LTE) radio interface to communicate with the serving BS and a group of users within its coverage. A MFemtocell and its associated users are all viewed as a single unit to the BS.

The implementation of MFemtocell can potentially benefit cellular systems. First of all, MFemtocells can improve the spectral efficiency of the entire network. MFemtocells can also contribute to signaling overhead reduction in the network for improving the system performance. For instance, MFemtocell can perform a handover on behalf of all its associated users. This will reduce the handover attempts as the users move between cells in the network. Furthermore, due to a relatively shorter range communication with their serving MFemtocell, the battery life of the associated users can be prolonged. It is also important to note that MFemtocells are located inside vehicles with antennas located outside the vehicle. This setup improves the signal quality inside the vehicle. The MFemtocell implementation can be backward compatible with the legacy handsets and the network. It may not require any changes in the Radio Access Network (RAN). The MFetmocell traffic over the air interface is treated as a single user equipment (UE) traffic. Therefore, a MFemtocell is actually a transceiver similar to a UE but with more advanced capabilities. For example, a larger number of antennas can be utilized at the MFemtocell with significant diversity/multiplexing gain. In addition, the users handover from the serving macrocell to the MFemtocell and vice versa since they see the latter as a regular BS or more precisely a Femtocell station.

\section{System MOdel AND SPECTRUM ANALYSis}

Let us consider a single cell with multiple MFemtocells and multiple users, as depicted in Fig. 1. We focus on MFemtocell-assisted LTE downlink multiuser communications in this model. For convenience, the macrocell users, which communicate directly with the eNodB, and MFemtocells are indexed by $n \in \mathcal{N}=\{1, \ldots, N\}$ and $j \in \mathcal{J}=\{1, \ldots, J\}$, respectively. For any MFemtocell $j(j \in \mathcal{J})$, there is a set of active users $m \in \mathcal{M}_{j}=\{1, \ldots, M\}$. Note that the total number of end users is $N$ including the users within MFemotocells. A user within a MFemtocell will be indexed by its respective $n$ if it communicates directly with the eNodB. The eNodB and all MFemtocell transmit with fixed power per Resource Block (RB). To support the opportunistic scheduling, the eNodB gathers the channel state information (CSI) from all users and MFemtocells. Likewise, the users within a MFemtocell will feed back this information but to the MFemtocell only. The terms backhaul link, access link, and direct link are used to denote eNodB-MFemtocell, MFemtocell-user, and eNodBuser links, respectively. The eNodB will transmit data to
MFemtocells over backhaul links. A MFemtocell will then fully decode, buffer, and re-transmit the data to its user (Decode-and-Forward multi-hop strategy). We assume that the backhaul, access, and direct links all experience Non-lineof-sight (NLoS) Rayleigh block fading channels, which are kept constant within a sub-frame and change independently in the following sub-frame. We also assume that the backhaul link has a gain $(G)$ over the direct link. This gain can be achieved by using a highly directional antenna pattern as well as pointing MFemtocell's antenna towards the eNodB. Practically, the multi-hop device cannot transmit and receive data simultaneously on the same frequency. Therefore, a division scheme is required, either in the time or frequency domain, to prevent the self interference. In this paper, time division is assumed.

\section{A. Resource Partitioning Schemes}

In an OFDMA-based cellular system, the whole spectrum is split into orthogonal sub-channels. These sub-channels are shared by different users by means of opportunistic resource allocation. By bringing in the MFemtocell, the spectrum has to be allocated (or reused) among different links, i.e., the backhaul, direct and access links. Therefore, it is essential to design an efficient resource partitioning policy in the MFemtocell-enhanced system to improve the performance of the whole system and limit the intra-cell interferences. Two resource partitioning policies can be used and are explained as follows:

1) Orthogonal resource partitioning scheme: In this scheme, the radio resources allocated to the backhaul, direct, and access links are all orthogonal either in the time or frequency domain and hence there is no intra-cell interference from the the eNodB to MFemtocell users and vice versa. Therefore, the Signal-to-Noise Ratios (SNRs) for a direct user, $\mathrm{SNR}_{(\mathrm{D})}$, and an access user, $\mathrm{SNR}_{(\mathrm{A})}$, can be calculated by $\mathrm{SNR}_{n_{(\mathrm{D})}}=\frac{P_{1}\left|h_{n}^{\mathrm{eNB}}\right|^{2}}{B N_{o}}$ and $\mathrm{SNR}_{m_{(\mathrm{A})}}=\frac{P_{2}\left|h_{m}^{j}\right|^{2}}{B N_{o}}$, respectively, where $h_{n}^{\mathrm{eNB}}$ and $h_{m}^{j}$ are complex-valued channel gains over the direct link and access link, respectively, and $P_{2}$ is the MFemtocell transmission power. $B$ and $N_{o}$ are the system bandwidth and the noise spectral density, respectively,

2) Non-orthogonal resource partitioning scheme: In this scheme, the radio resources are reused by the direct and access links. However, the radio resources are still orthogonally allocated between backhaul and direct and between backhaul and access links. Non-orthogonal mode means that there will be an intra-cell interference to the access and direct users due to the simultaneous transmissions from the MFemtocell and eNodB on the same sub-channels. The advantage of this scheme is the improvement in resource utilization compared to the orthogonal scheme. In addition, this scheme gives the flexibility to implement Radio Resource Management (RRM) at the eNodB and the MFemtocell independently. The received Signal to Interference-plus-Noise Ratio (SINR) for a direct user, denoted as $\operatorname{SINR}_{(\mathrm{D})}$, can be calculated by $\operatorname{SINR}_{n_{(\mathrm{D})}}=\frac{P_{1}\left|h_{n}^{\mathrm{eNB}}\right|^{2}}{I+B N_{o}}$ where $I$ is the intra-cell interference from the MFemtocells. This type 
of interference can be reduced significantly by constraining the transmission power within the MFemtocell using a directive antenna. On the other hand, the received SINR for an access link user, $\operatorname{SINR}_{(\mathrm{A})}$, is given by:

$$
\begin{aligned}
\operatorname{SINR}_{m_{(\mathrm{A})}} & =\frac{P_{2}\left|h_{m}^{j}\right|^{2}}{\left|h_{n}^{\mathrm{eNB}}\right|^{2}+B N_{o}} \\
& =\frac{\operatorname{SNR}_{m_{(\mathrm{A})}}\left|h_{m}^{j}\right|^{2}}{\operatorname{SINR}_{n_{(\mathrm{D})}}\left|h_{n}^{\mathrm{eNB}}\right|^{2}+1} .
\end{aligned}
$$

So, the $\mathrm{SINR}_{m_{(\mathrm{A})}}$ in (1) can be characterized by SNR received from a MFemtocell, i.e., $\mathrm{SNR}_{m_{(\mathrm{A})}}$, and $\mathrm{SNR}$ received from the eNodB if the same user was served by eNodB instead, i.e., $\mathrm{SNR}_{n_{\text {(D) }}}$. Now, if we assume that the distances from the MFemtocell or its users are the same with respect to the eNodB, we have $\operatorname{SINR}_{m_{(\mathrm{A})}}=\frac{\mathrm{SNR}_{m_{(\mathrm{A})}}\left|h_{m}^{j}\right|^{2}}{\frac{\mathrm{SNR}_{j_{(\mathrm{B})}}}{G}\left|h_{n}^{\mathrm{eNB}}\right|^{2}+1}$. The $\mathrm{SNR}_{j_{(\mathrm{B})}}$ is the SNR for a backhaul channel for MFemtocell $j$ which can be calculated, for both resource partitioning schemes, by $\mathrm{SNR}_{j_{(\mathrm{B})}}=\frac{P_{1}\left|h_{j}^{\mathrm{eNB}}\right|^{2} G}{B N_{o}}$ where $\left|h_{j}^{\mathrm{eNB}}\right|^{2}$ is the channel power gain of the backhaul link for MFemtocell $j$. The interference that originates from the eNodB can be compromised with good propagation quality between the MFemtocell and its users due to shorter range transmissions.

\section{B. MFemtocell Spectrum Reuse}

Another form of spectrum reuse scheme can be applied to MFemtocell scenarios to further improve the spectrum utilization. Hence, multiple MFemtocells can use a common set of sub-channels simultaneously to serve their users. However, this can work only if multiple MFemtocells are located large distances apart or the coverage of each MFemtocell is limited to a small area by using a directive antenna.

\section{Spectral Efficiency Analysis}

Multiuser scheduling is assumed here where the macrocell users and MFemtocells are served over $K \mathrm{RBs,} \mathrm{indexed}$ by $k=1, \ldots, K$, based on the well-known MAX-SINR and Proportional Fair (PF) scheduling policies. With the MAXSINR scheduler, the eNodB will assign a RB $k$ to a user $n$ having the highest instantaneous SINR at a sub-frame $t$. Thus, arg $\max _{n \in \mathcal{N}} R_{n}(t, k), \quad k=1, \ldots, K$, where $R_{n}(t, k)$ $\propto \operatorname{SNR}_{n_{(\mathrm{D})}}(t, k)$ (or $\operatorname{SINR}_{n_{(\mathrm{D})}}(t, k)$ in non-orthogonal mode) is the instantaneous achievable rate on $\mathrm{RB} k$ for a user $n$ and is calculated according to the following Shannon formula. In the PF scheduling case, the scheduler allocates the $\mathrm{RB} k$ to a user $n \in \mathcal{N}$ according to the following criterion:

$$
\bar{n}_{k}=\arg \max _{n \in \mathcal{N}} \frac{R_{n}(t, k)}{\bar{R}_{n}(t)} \quad k=1, \ldots, K
$$

where $\bar{R}_{n}(t)$ is the average delivered rate in the past, measured over a fixed window of observation. It can be calculated using an average filtering [15], which will be updated using the following formula:

$$
\bar{R}_{n}(t)=\left(1-\frac{1}{T}\right) \bar{R}_{n}(t-1)+\frac{1}{T} \sum_{k=1}^{K} R_{n}(k, t) d_{n}(k, t)
$$

where $T$ is the time window constant, $d_{n}(k, t)$ is a binary indicator that is set to 1 if the user $n$ is scheduled on resource block $k$ at time $t$ and to 0 otherwise.

The communication over the eNodB-MFemtocell links takes place over a dedicated time-frequency zone, as shown in Fig. 2(a) and Fig. 2(b). Moreover, a set of MFemtocells is selected based on the same scheduling algorithm that has been used to serve macrocell users. Within the MFemtocell, it is assumed that the users $\left(\mathcal{M}_{j}\right)$ are served according to roundrobin policy. In case of the orthogonal scheme, it is assumed that a fraction of the spectrum $\beta, 0<\beta<1$, is allocated exclusively for direct transmissions in the second portion of the time, as shown in Fig. 4(a). Whereas in the non-orthogonal scheme, the eNodB and MFemtocells can utilize the whole spectrum to serve their users, as shown in Fig. 4(b).

The achievable capacity (in bps/Hz/cell) on the direct link on time $t$ can be calculated by

$$
C_{\mathrm{d}}(t)=\left\{\begin{array}{lc}
\frac{\beta}{2 B} \sum_{n=1}^{N} \sum_{k=1}^{K} R_{n}(k, t) d_{n}(k, t) & \text { orthogonal } \\
\frac{1}{2 B} \sum_{n=1}^{N} \sum_{k=1}^{K} R_{n}(k, t) d_{n}(k, t) & \text { non orthogonal. }
\end{array}\right.
$$

The achievable capacity on the access link can be given by

$$
C_{2}^{j}(t)=\left\{\begin{array}{l}
\frac{(1-\beta)}{2 B} \sum_{m \in \mathcal{M}_{j}} \sum_{k=1}^{K} R_{m}(k, t) d_{m}(k, t) \quad \text { orthogonal } \\
\frac{1}{2 B} \sum_{m \in \mathcal{M}_{j}} \sum_{k=1}^{K} R_{m}(k, t) d_{m}(k, t) \text { non orthogonal }
\end{array}\right.
$$

where $R_{m}(t, k) \propto \operatorname{SNR}_{m_{(\mathrm{A})}}(t, k)$ is the instantaneous achievable rate for an access user $m$. However, the rates on access link between MFemtocells and their users are truncated by the achievable capacity of the backhaul link for MFemtocell $j$, i.e.,

$$
C_{1}^{j}(t)=\frac{1}{2 B} \sum_{k=1}^{K} R_{j}(k, t) d_{j}(k, t) .
$$

where $R_{j}(t, k)$ is the instantaneous achievable rate over the backhaul link for an MFemtocell $j$. As a result, the total system capacity (in bps/Hz/cell) after allocating all RBs to the selected users, including MFemtocell users, can be calculated according to:

$$
C_{s y s}(t)=\sum_{j=1}^{J} \min \left[C_{1}^{j}(t-d), C_{2}^{j}(t)\right]+C_{\mathrm{d}}(t)
$$

where $d$ is the time required to decode, buffer, and re-encode the incoming data from the backhaul links. The first term in (7) stands for the achievable capacity of data flow from eNodB to users via MFemtocell and the second term represents the capacity for direct users. It is worth mentioning that the throughput on the backhaul links is not accounted for system capacity because the data are not delivered to the users, but it still bounds the throughput on the access links. However, to 
get an efficient resource usage over the multi-hop, the rates over the backhaul and access should be equal, i.e., $C_{1}=C_{2}$.

\section{Simulation Results and Discussions}

The performance of the MFemtocell in the LTE system was evaluated using a dynamic system level simulator which is compliant with 3GPP LTE specification [16]. The simulation consists of several iterations and each of them has 20000 subframes. In each iteration, the MFemtocells and mobile users were distributed independently. A frequency-selective channel model with 6 taps and a certain power delay profile (PDP) were considered. The number of MFemtocell and macrocell users were assumed to be 10 and 40, respectively. Inside each MFemtocell, it was assumed that there were two active users. The LTE frame structure was considered, which consists of blocks of 12 contiguous sub-carriers in the frequency domain and 7 OFDM symbols in the time domain. One subframe $(1 \mathrm{~ms})$ was regarded as the scheduling period. The carrier bandwidth was fixed at $10 \mathrm{MHz}$ with $50 \mathrm{RBs}$. All UEs were equipped with a single antenna while the MFemtocells have two antennas working in the diversity mode. The gain $(G)$ was assumed to be $8 \mathrm{~dB}$. A full eNodB buffer was considered where there were always buffered data ready for transmission for each node. The users inside the MFemtocell were assumed to be indoor users with $5 \mathrm{~dB}$ penetration loss. Other relevant simulation parameters are summarized in Table 1.

Fig. 3 compares the average spectral efficiencies of the orthogonal and non-orthogonal schemes in MFemtocellenhanced scenarios as functions of the percentage of users that associate with MFemtocells. As it is clearly shown, increasing the percentage of users that communicate with an eNodB through the MFemtocell leads to an increase in the spectral efficiency compared with a single-hop reference system. The simulation results also demonstrate that the non-orthogonal scheme outperforms the orthogonal scheme due to spectrum reuse between the eNodB and the MFemtocells.

The Cumulative Distribution Functions (CDFs) of the user and MFemtocell average throughputs are presented in Fig. 4, where only the non-orthogonal scheme and PF scheduling are assumed. It is clear that the MFemtocell deployment can increase the average throughput of macro users. Furthermore, the MFemtocells have high throughputs due to the additional gain in received SINR $_{(\mathrm{B})}$ on the backhaul link.

Fig. 5 depicts the system throughputs of the two partitioning schemes as functions of $\mathrm{SNR}_{(\mathrm{D})}$ of the direct link on different cell positions. Here, it has been assumed that all the RBs are allocated to only two users: one connected directly to the eNodB and the other through the MFemtocell. Both schemes provide a better performance, as compared to the single-hop reference system, especially at the middle and edge of the cell. However, when the MFemtocells are moving near the eNodB, i.e., when $\mathrm{SNR}_{(\mathrm{D})}$ is larger than $14 \mathrm{~dB}$, the performance of the orthogonal scheme lags slightly behind that of the single hop scheme due to the high SNR on the direct link. In this case, it is worth transmitting directly to users.

\section{CONCLUSIONS}

In this paper, we have introduced the architecture of MFemtocell which can be a potential candidate technology for LTE-Advanced systems. We have studied the performance of two resource partitioning schemes which can be used for MFemtocell deployed scenarios in LTE-Advanced systems in presence of opportunistic scheduling. Our system-level simulations have shown that the performance of a cellular system with MFemtocell implementation outperforms that of a system without MFemtocell, especially at the middle and edge of the cell. The results have also demonstrated that the nonorthogonal scheme can achieve better performance compared with the orthogonal scheme.

\section{ACKNOWLEDGMENTS}

This work is partially supported by Hutchison $3 \mathrm{G}$ in the UK. The authors would like to acknowledge the support from the RCUK for the UK-China Science Bridges Project: R\&D on (B) $4 \mathrm{G}$ Wireless Mobile Communications. F. Haider, C.-X. Wang, and H. Haas would also like to acknowledge the support from the Scottish Funding Council for the Joint Research Institute in Signal and Image Processing between the University of Edinburgh and Heriot-Watt University, as part of the Edinburgh Research Partnership in Engineering and Mathematics (ERPem). The work of C.-X. Wang was also supported by the Opening Project of the Key Laboratory of Cognitive Radio and Information Processing (Guilin University of Electronic Technology), Ministry of Education, under Grant 2009KF02.

\section{REFERENCES}

[1] O. Oyman, N. Laneman, and S. Sandhu, "Multihop relaying for broadband wireless mesh networks: from theory to practice," IEEE Commun. Magazine, vol. 45, no. 11, pp. 116-122, Nov. 2007.

[2] C.-X. Wang, X. Hong, X. Ge, X. Cheng, G. Zhang, and J. Thompson, "Cooperative MIMO channel models: a survey," IEEE Commun. Magazine, vol. 48, no. 2, pp. 80-87, Feb. 2010.

[3] X. Hong, C.-X. Wang, M. Uysal, X. Ge, and S. Ouyang, "Capacity analysis of hybrid cognitive radio networks with distributed VAAs," IEEE Trans. Veh. Techno., vol. 59, no. 7, pp. 3510-3523, Sept. 2010.

[4] R. Pabst, B. Walke, D. Schultz, P. Herhold, H. Yanikomeroglu, S. Mukherjee, H. Viswanathan, M. Lott, W. Zirwas, M. Dohler, H. Aghvami, D. Falconer, and G. Fettweis, "Relay-based deployment concepts for wireless and mobile broadband radio," IEEE Commun. Magazine, vol. 42, no. 9, pp. 80-89, Sept. 2004.

[5] D. Soldani and S. Dixit, "Wireless relays for broadband access," IEEE Commun. Magazine, vol. 46, no. 3, pp. 58-66, Mar. 2008.

[6] M. Kaneko and P. Popovski, "Adaptive resource allocation in cellular OFDMA system with multiple relay stations," in Proc. IEEE VTC07Spring, Apr. 2007, pp. 3026-3030.

[7] O. Oyman, Opportunistic scheduling and spectrum reuse in relay-based cellular networks," IEEE Trans. Wireless Commun., vol. 9, no. 3, pp. 1074-1085, Mar. 2010.

[8] H. Nourizadeh, S. Nourizadeh, and R. Tafazolli, "Performance evaluation of cellular networks with mobile and fixed relay station," in Proc. IEEE VTC06-Fall, Sept. 2006, pp. 1-5.

[9] L. Xiao, T. Fuja, and D. Costello, "An analysis of mobile relaying for coverage extension," in Proc. IEEE Symp. Info. Theory, July 2008, pp. 2262-2266.

[10] 3GPP, TR 36.921 v.9.0.0,"Evolved Universal Terrestrial Radio Access (E-UTRA); FDD Home eNode B (HeNB) Radio Frequency (RF) requirements analysis", Rel. 9, Apr. 2010.

[11] V. Chandrasekhar, J. Andrews, and A. Gatherer, "Femtocell networks: a survey," IEEE Commun. Magazine, vol. 46, no. 9, pp. 59-67, Sept. 2008.

[12] H. Claussen, "Performance of macro- and co-channel Femtocells in a hierarchical cell structure," in Proc. IEEE PIMRC07, Sept. 2007, pp. 1-5. 
TABLE I

SIMULATION PARAMETERS.

\begin{tabular}{c|c|c|c} 
Parameter & eNodB-MFmeto & eNodB-UE & MFemto-UE \\
\hline Antenna height $(\mathrm{m})$ & 20 & 20 & 2 \\
Shadwoing $(\mathrm{dB})$ & 8 & 8 & 4 \\
Antenna gain $(\mathrm{dBi})$ & 18 & 18 & 5 \\
Bandwidth $(\mathrm{MHz})$ & 10 & 10 & 10 \\
Transmitted power $(\mathrm{dBm})$ & 46 & 46 & 20 \\
Spectrum sharing $(\beta)$ & $100 \%$ & $50 \%$ & $50 \%$ \\
Distance $(\mathrm{m})$ & $\geq 100$ & $\geq 100$ & $\leq 15$ \\
\hline
\end{tabular}

[13] I. Guvenc, M.-R. Jeong, F. Watanabe, and H. Inamura, "A hybrid frequency assignment for Femtocells and coverage area analysis for cochannel operation," IEEE Commun. Letters, vol. 12, no. 12, pp. 880-882, Dec. 2008.

[14] Y. Bai, J. Zhou, and L. Chen, "Hybrid spectrum usage for overlaying lte macrocell and Femtocell," in Proc. IEEE GLOBECOMO9, Dec. 2009, pp. 1-6.

[15] A. Jalali, R. Padovani, and R. Pankaj, "Data throughput of CDMA-HDR a high efficiency-high data rate personal communication wireless system," in Proc. IEEE VTCO0-Spring, May 2000, pp. 1854-1858.

[16] 3GPP, TR 25.814 V7.1.0, "Physical Layer Aspects for Evolved UTRA," Rel. 7, Sep. 2006.

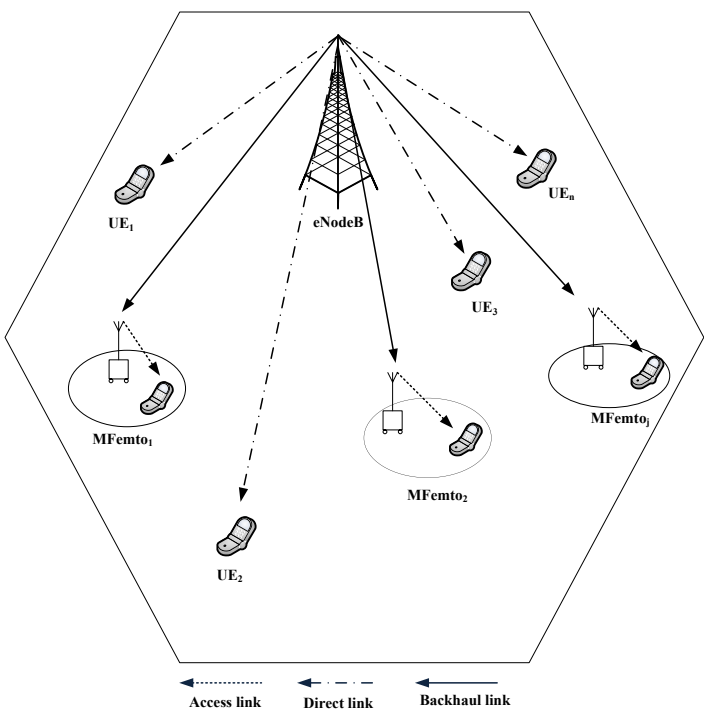

Fig. 1. System model: a single cell with multiple MFemtocells and users.
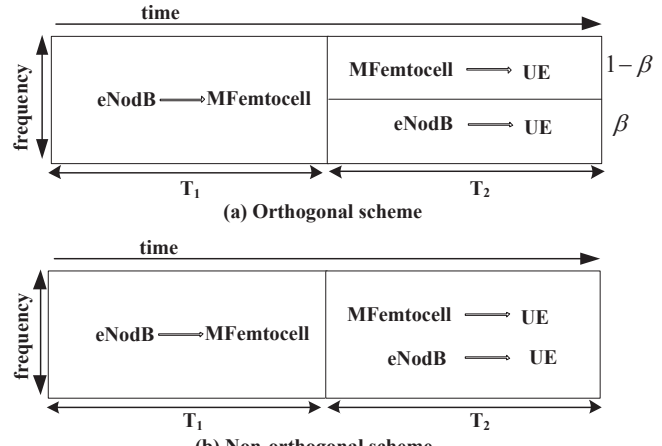

(b) Non-orthogonal scheme

Fig. 2. Resource partitioning schemes.

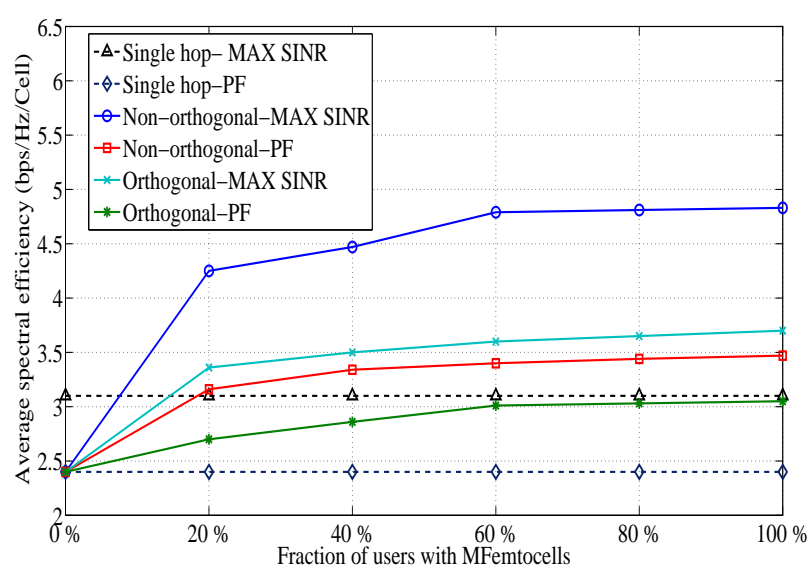

Fig. 3. Spectral efficiency of system-level MFemtocells with multiuser scheduling and resource partitioning schemes.

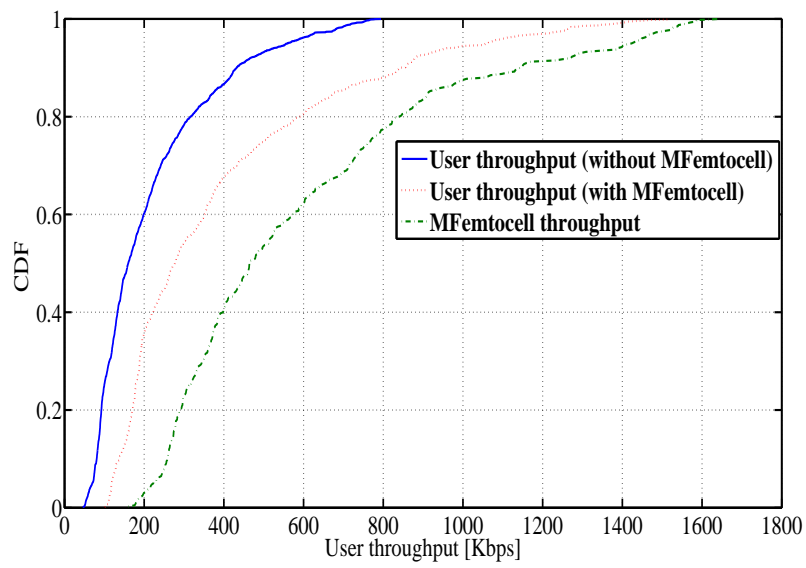

Fig. 4. The CDFs of user \& MFemtocell average throughputs.

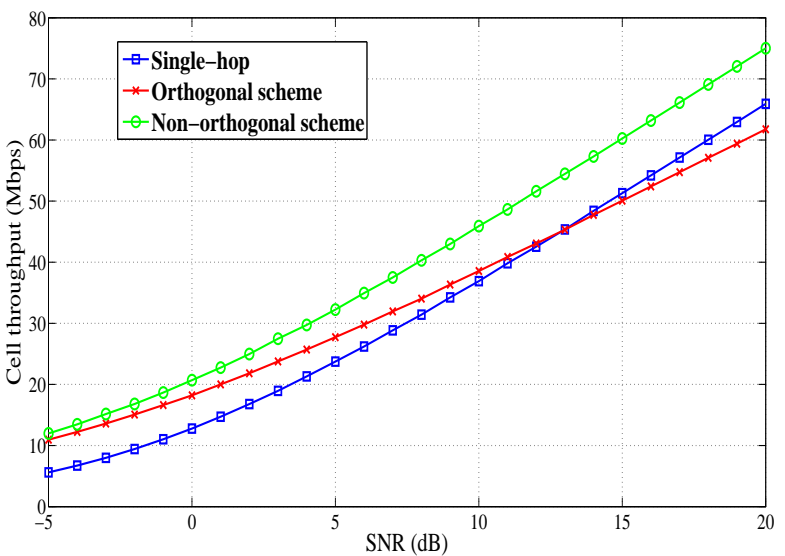

Fig. 5. Cell throughput performance as a function of $\mathrm{SNR}_{(\mathrm{D})}$. 